\title{
Categorizing pain and distress in a Parkinson's model
}

Parkinson's disease is a neurological disorder usually diagnosed in persons over 65 years of age. Some of the symptoms include limb tremors, slow movement, and loss of balance. Dr. Golda Sherman studied the disease using an adult rhesus monkey model. Her basic procedure was to give regular subcutaneous injections of the neurotoxin N-methyl-4-phenyl1,2,3,6-tetrahydropyridine (MPTP) over a period of at least 4 months. Then, after a stabilization period, Sherman evaluated the efficacy of various drugs for treating the animals. Many of the clinical signs of Parkinson's in the animals mimic those seen in humans. More often than not the monkeys required assistance with feeding, and this help was routinely provided by the research group. Recently, in collaboration with colleagues at another university, Sherman developed a research interest in using adeno-associated virus-based vectors (AAV2) that encode a therapeutic human gene as a potential treatment for animals with Parkinson's.

Sherman's new IACUC protocol had three study groups: (1) MPTP only, (2) MPTP plus AAV2 without the gene of interest, and (3) MPTP plus AAV2 with the gene. The protocol called for the latter two groups to have the AAV2 injected intracranially using stereotaxic surgery. These AAV2-treated animals were to be placed in USDA pain/distress Category $\mathrm{D}$ (pain or distress alleviated by drugs) because of the surgery. The MPTP-only animals were to be placed in Category C (no pain or distress), the same category in which Great Eastern University had previously approved Sherman's studies. The protocol went through veterinary 'pre-review' without incident, and was then presented to the IACUC.

At the full committee meeting one of the newer members raised two questions. First, if an animal was going to develop signs of Parkinson's disease, why place it in Category C rather than Category E (unalleviated pain or distress)? Further, why not consider the animals undergoing surgery as part of multiple major operative procedures? Malcolm Michaels, the IACUC Chairman, explained that because Sherman provided the non-surgical animals with adequate medical and husbandry attention, Category $\mathrm{C}$ was appropriate for them. Moreover, because only one invasive surgical procedure was being done on the remaining animals, there was no need to consider this study as encompassing multiple major operative procedures.

Do you agree with the IACUC's determination that this is a Category $\mathrm{C}$ study for the non-surgical monkeys and Category D for the others? Should the IACUC consider Sherman's research as multiple major operative procedures on the same animal, or is it a single major operative procedure?

\section{RESPONSE}

\section{Gimme an ' $E$ '}

\section{Ellen M. Levee, DVM}

The Great Eastern IACUC underwent an experience familiar to anyone that has served on an IACUC for any length of time. New members bring a new insight and interpretation to longstanding protocols. This 'new blood' can help the entire Committee and the investigator to reexamine their classification of the protocols with respect to pain and distress categories or other issues. The IACUC Chair should certainly nurture discussions of this nature and not dismiss the types of inquiries raised regarding this protocol. The fact that nonhuman primates are involved in a study as an induced model of Parkinson's disease furthers the need for careful and extensive review of the protocol.

This protocol should place all animals in the same category since they all undergo induction of a model of Parkinson's disease by MPTP. The category of pain or distress should be E (unalleviated pain or distress). This model is certainly associated with a degree of unalleviated distress since the animals require additional medical and husbandry assistance. Although the animals are given the additional attention, there is distress intrinsic to the model that is not eliminated by providing the necessary care. The surgically manipulated monkeys would still fall under this category since they initially are induced with MPTP to create the model.

The IACUC Chair was correct to state that the surgical procedure was a single major operative procedure as opposed to multiple survival surgeries on a single animal. The surgically manipulated animals undergo a single stereotaxic surgery in order to deliver the AAV2 intracranially. If additional intracranial injections are required, a permanent port could be placed at the time of stereotaxic surgery that would facilitate injections without additional surgeries.

Levee is Director, Department of Comparative Medicine, and Assistant Professor, Departments of Physiology and Pathology, New York Medical College, Valhalla, NY. 